\title{
Remotely Powered and Reconfigured Quasi-Passive Reconfigurable Nodes for Optical Access Networks
}

\author{
Yingying Bi, ${ }^{1}$ Shunrong Shen, ${ }^{1}$ Jing Jin, ${ }^{1}$ Ke Wang, ${ }^{1,2,3}$ and Leonid G. Kazovsky ${ }^{1}$ \\ ${ }^{1}$ Department of Electrical Engineering, Stanford University, Stanford, CA 94305, USA \\ ${ }^{2}$ Centre for Neural Engineering (CfNE), University of Melbourne, Melbourne, VIC 3010, Australia \\ ${ }^{3}$ Department of Electrical and Electronic Engineering, University of Melbourne, Melbourne, VIC 3010, Australia
}

Correspondence should be addressed to Yingying Bi; yybi@stanford.edu

Received 25 November 2015; Accepted 24 January 2016

Academic Editor: Iraj Sadegh Amiri

Copyright (C) 2016 Yingying Bi et al. This is an open access article distributed under the Creative Commons Attribution License, which permits unrestricted use, distribution, and reproduction in any medium, provided the original work is properly cited.

\begin{abstract}
Quasi-Passive Reconfigurable (QPAR) nodes have been proposed to provide flexible power/wavelength allocation in optical access networks. QPAR only consumes power during reconfiguration, which is remotely transmitted from the central office, thus maintaining the passive nature of the network. In this paper, a QPAR control circuit is designed, and a remotely powered and reconfigured $1 \times 2 \times 2$ QPAR (i.e., one wavelength, two power levels, and two output ports) with a $0.1 \mathrm{~F} / 5 \mathrm{~V}$ supercapacitor (SC) remotely charged by a $1 \times 8$ photodiode array is experimentally demonstrated. The charged SC can power the QPAR for at least $6 \mathrm{~s}$ with 24 consecutive reconfigurations ( $200 \mathrm{~ms}$ each) or two reconfigurations within a maximum period of 40 hours, before the SC needs to be recharged. In addition, the demonstrated QPAR remote power scheme is compared with the previously proposed Direct Photovoltaic Power option both theoretically and experimentally. Results show that the SC based remote power mechanism is capable of driving a large number of reconfigurations simultaneously and it is better for large dimension QPARs.
\end{abstract}

\section{Introduction}

Passive Optical Networks (PONs) have been widely deployed in optical access networks. However, current PONs have the limitations of inflexible power/wavelength allocation due to the use of static passive components such as fixed power splitters and wavelength division multiplexing (WDM) couplers. Next-generation optical access networks should have sufficient intelligence and reconfigurability to manage different power and bandwidth requirements without sacrificing energy efficiency. Therefore, the Quasi-Passive and Reconfigurable (QPAR) node has been proposed in our previous studies [1]. The QPAR node can combine the following functions simultaneously in a quasi-passive manner in future PONs: (1) splitting the input power into adjustable levels and assigning them to different optical network units (ONUs) according to the ONUs' distances from the central office (CO); (2) dynamically allocating wavelengths to different groups of outputs based on user-specific bandwidth requirements. Another important advantage of QPAR is that it is quasi-passive; that is, it only requires power during reconfiguration with no steady-state power consumption.

The QPAR has been experimentally demonstrated using discrete components including $2 \times 2$ optical latching switches (OLSs), $3 \mathrm{~dB}$ couplers, and WDM couplers [1]. OLSs are the key components to realize the quasi-passive feature since it only consumes power during reconfiguration, and Microelectromechanical Systems- (MEMS-) based OLSs have been utilized $[1,2]$. The $2 \times 2$ MEMS-OLS can be driven by a $2.5 \mathrm{~V}-$ $5 \mathrm{~V}$ electrical signal, and a control logic is also needed to select between the bar/cross states. As a branching device in the remote node $(\mathrm{RN})$, the QPAR can be remotely powered by transmitting optical power from the $\mathrm{CO}$ through the feeder fiber [3]. This avoids on-site maintenance and configuration at the RN. Moreover, without having any local power supply, the passive nature of the $\mathrm{RN}$ is maintained.

One straightforward way of QPAR remote power is transmitting high power light to the $\mathrm{RN}$ during reconfigurations. At the RN, the power light is converted into electrical power by a photovoltaic power converter (PPC), which is used 
to power the QPAR and its control circuit directly. This Direct Photovoltaic Power scheme has been proposed and demonstrated in our previous studies [3]. An eight-in-series InGaAs PD array was used to convert the optical power at $1480 \mathrm{~nm}$ into the electrical power for QPAR reconfiguration. In this design, every time QPAR needs to be reconfigured, it is directly powered with the PD array. The reconfiguration of QPAR is initiated and ended by turning on and off the high power laser in the CO. Experimental results have shown that a minimum $4 \mathrm{dBm}$ optical feed into each $\mathrm{PD}$ is required to power one $2 \times 2 \mathrm{OLS}$, while $9 \mathrm{dBm}$ is required to power two OLSs in parallel.

Direct Photovoltaic Power option is a promising solution to power a small dimension QPAR. However, for large dimension QPARs, this option requires a high instantaneous power from the PPC [3] or multiple PPCs with multiple wavelengths [4], thus lacking scalability. To overcome these limitations, there is another option, storing the energy in a supercapacitor, which has been proposed to power switching units in metro or access networks, for example, an electrooptic $2 \times 2$ switch [5]. However, a systematic comparison between the two options, that is, the Direct Photovoltaic Power option and Charged Supercapacitor Power option, is still missing.

In this paper, a QPAR remote power mechanism using energy stored in an optically charged supercapacitor, that is, the Charged Supercapacitor Power option, is proposed, investigated, and experimentally demonstrated. Furthermore, the Direct Photovoltaic Power and Charged Supercapacitor schemes for QPAR remote power are compared both theoretically and experimentally. In addition, a control circuit that can be used to reconfigure QPAR states is designed and demonstrated with remote power.

The rest of paper is organized as follows. Section 2 presents the principle of Charged Supercapacitor Power scheme for QPAR remote power. In Section 3, two QPAR remote power mechanisms are analyzed and compared. Section 4 shows the designed control circuit for QPAR remote reconfiguration, and the experimental demonstration of a QPAR node using the designed remote power system and control circuit. Conclusions of this paper are given in Section 5 .

\section{Charged Supercapacitor Power of QPAR}

The QPARs can be remotely powered using the supercapacitor (SC), which is also known as ultracapacitor. Different from a regular capacitor, the SC has a much larger capacitance, which is often rated in farads [6]. Therefore, SCs typically store 10 to 100 times more energy per unit volume than electrolytic capacitors. Compared with rechargeable batteries, SCs can accept and deliver charges more rapidly, tolerate more charging and discharging cycles, and have fewer safety issues. Therefore, using charged SC is an attractive choice for QPAR remote power.

The proposed general architecture of the QPAR remote power system using a SC is shown in Figure 1. In this design, when reconfigured, QPAR is powered by the SC in the RN, which is charged by the remote power system optically. Unlike the Direct Photovoltaic Power option, the power and control signals of the QPAR node are not transmitted simultaneously. The high power laser is turned on only during the SC charging process, while the control signal is generated and transmitted during the discharging process for QPAR reconfiguration. To avoid current leakage that decelerates charging, an analog switch is used to isolate the load from the SC during the charging process. Therefore, the reconfiguration process is initiated and ended by switching on and off the analog switch.

2.1. Charging of the Supercapacitor. In our proposed QPAR remote power system, the SC was charged with a PPC (PPC1 as shown in Figure 1). The PPC was a $1 \times 8 \mathrm{InGaAs} \mathrm{PD}$ array, and its IV curves under different optical power conditions are plotted in Figure 2. A 0.1 F/5 V SC was used and the charging curve was measured as shown in Figure 3. According to Figure 3, the charging process of the SC with the PD array can be divided into two stages. The first stage is constant current charging, where the voltage on the SC increases linearly with time. The second stage is saturation charging, where the voltage slowly approaches the open circuit voltage of the PD array as the photocurrent drops sharply. To save the charging time, the charging should be stopped before the second stage. According to Figure 3 , the charging stopping voltage $\left(V_{1}\right)$ is about $4.6 \mathrm{~V}$. The charging time $T_{\text {charge }}$ is then approximately

$$
T_{\text {charge }}=\frac{\left(C \cdot V_{1}\right)}{I_{\text {charge }}}, \quad I_{\text {charge }}=P_{\text {in }} \cdot R_{P},
$$

where $C, V_{1}, I_{\text {charge }}, P_{\text {in }}$, and $R_{P}$ are the SC capacitance, charging stopping voltage, charging current, input optical power of each PD, and responsivity of the PD.

The charging time, which is the time it takes for the SC to be charged from $0 \mathrm{~V}$ to $4.6 \mathrm{~V}$, was measured and shown in Figure 4. It is clear that a higher feed power is desired for faster charging; however, the responsivity of the PDs decreases as the input light intensity increases due to the photocurrent saturation, as shown in Figure 4. Therefore, the optical feed power of the PD array should be selected to achieve both reasonably high conversion efficiency and acceptable charging time. $4 \mathrm{dBm}$ optical feed per PD (i.e., $13 \mathrm{dBm}$ into the whole $\mathrm{PD}$ array), where the $\mathrm{PD}$ array has a high responsivity of $0.97 \mathrm{~A} / \mathrm{W}$, was chosen in our demonstration of the $1 \times 2 \times 2$ QPAR remote power (Section 4.3). It takes $310 \mathrm{~s}$ to charge the SC from $0 \mathrm{~V}$ to $4.6 \mathrm{~V}$.

Using the equivalent circuit model of the PPC with a capacitor load, the capacitor charging voltage as a function of time can be calculated theoretically through transient analysis [7]. Table 1 lists the theoretical charging time of the $0.1 \mathrm{~F} \mathrm{SC}$ with an eight-in-series ideal InGaAs PD array, together with the corresponding experimental results. It can be seen that the experimental values are higher and the difference becomes more significant as the optical feed increases due to the deteriorating photocurrent saturation.

2.2. Discharging of the Supercapacitor. When the fully charged SC is used to power the QPAR, a discharging stopping voltage, denoted as $V_{2}$, and the SC capacitance should be selected appropriately so that the reconfiguration of 


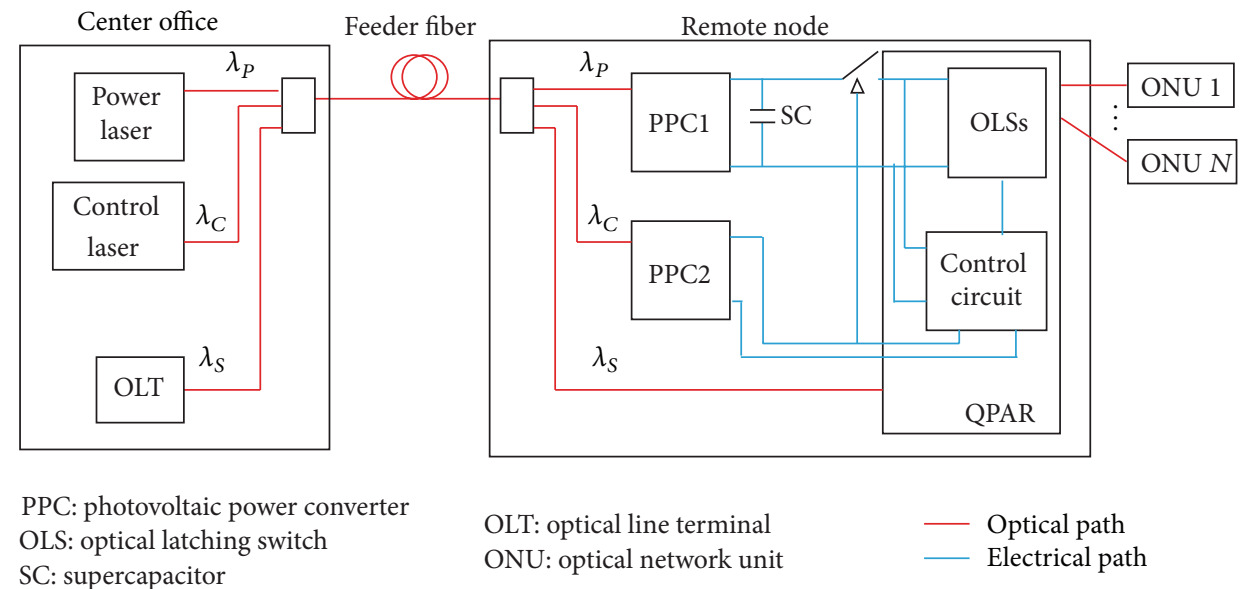

FIGURE 1: General architecture of the QPAR remote power system.

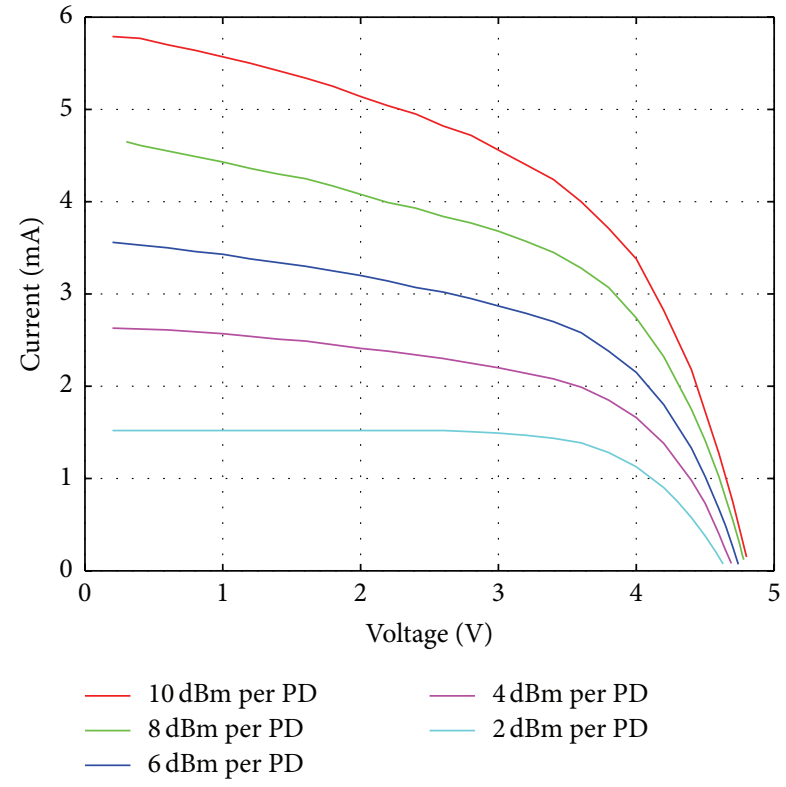

FIgURE 2: Experimental IV curves of the $1 \times 8 \mathrm{PD}$ array.

TABLE 1: Charging time of $0.1 \mathrm{~F} \mathrm{SC}$ (from $0 \mathrm{~V}$ to $4.6 \mathrm{~V}$ ).

\begin{tabular}{lcc}
\hline $\begin{array}{l}\text { Optical feed per } \\
\text { PD }\end{array}$ & $\begin{array}{c}\text { Theoretical } \\
\text { charging time }\end{array}$ & $\begin{array}{c}\text { Experimental } \\
\text { charging time }\end{array}$ \\
\hline $10 \mathrm{dBm}$ & $54 \mathrm{~s}$ & $143 \mathrm{~s}$ \\
$8 \mathrm{dBm}$ & $87 \mathrm{~s}$ & $179 \mathrm{~s}$ \\
$6 \mathrm{dBm}$ & $150 \mathrm{~s}$ & $230 \mathrm{~s}$ \\
$4 \mathrm{dBm}$ & $220 \mathrm{~s}$ & $310 \mathrm{~s}$ \\
\hline
\end{tabular}

QPAR can be stably completed before the SC voltage reaches $V_{2}$. The SC capacitance can be decided using

$$
P_{\mathrm{QPAR}} \cdot T_{\mathrm{rec}}=\frac{1}{2} C\left(V_{1}^{2}-V_{2}^{2}\right),
$$

where $P_{\mathrm{QPAR}}, T_{\text {rec }}, C, V_{1}$, and $V_{2}$ are the average power consumption of the QPAR node, reconfiguration time, the SC

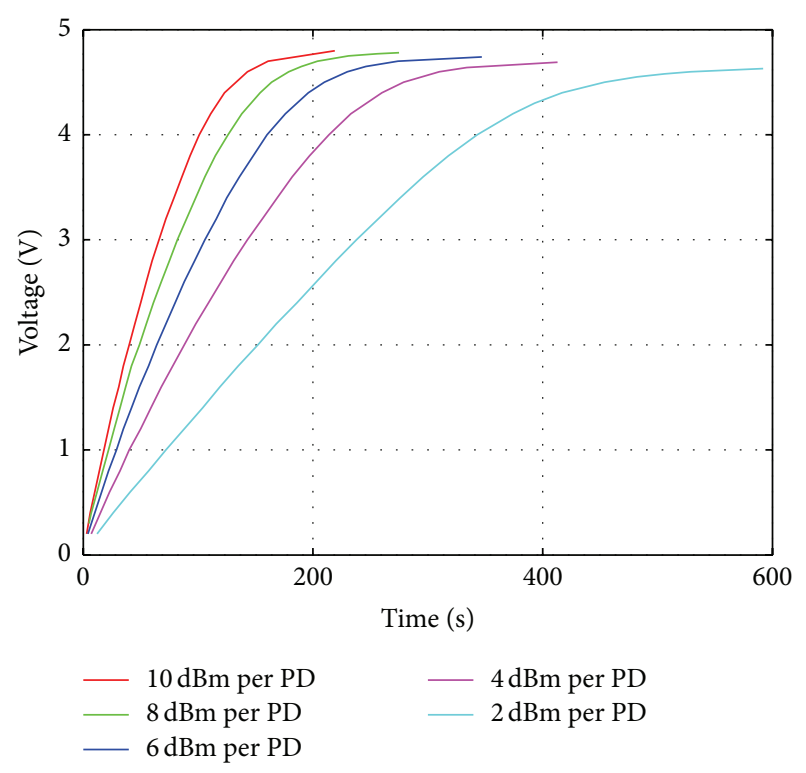

FIGURE 3: Experimental charging curves of a $0.1 \mathrm{~F} \mathrm{SC}$.

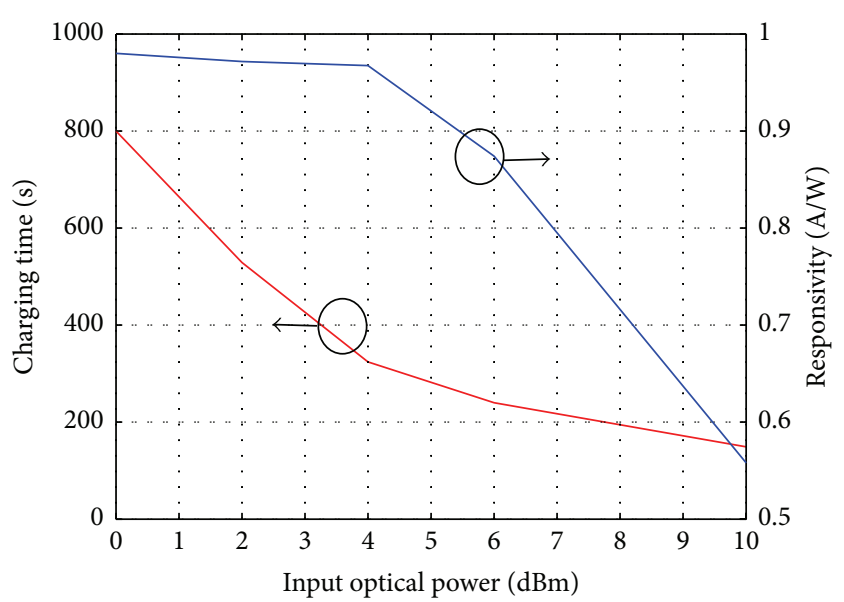

FIGURE 4: Trade-off between the charging time and responsivity. 
capacitance, and charging and discharging stopping voltages. The reconfiguration time $\left(T_{\text {rec }}\right)$ may vary in applications but should be longer than the minimum driven pulse width of the OLSs. For the $2 \times 2$ OLS used in experiments, the minimum pulse width is $10 \mathrm{~ms}$ according to the data sheets [8] and $0.23 \mathrm{~ms}$ in the measurements [3].

The consumed current as well as the power consumption of the OLS we used rises to a peak value when the voltage supply is close to $2.8 \mathrm{~V} \mathrm{[3].} \mathrm{As} \mathrm{a} \mathrm{result,} \mathrm{although} \mathrm{the} \mathrm{OLS} \mathrm{can}$ work at a lower voltage of $2.5 \mathrm{~V}$, the SC discharging stopping voltage was selected at $3.5 \mathrm{~V}$ to avoid the current peak. This voltage can be further reduced if a DC-DC converter is used to boost the SC voltage to a constant value. However, this expedites the discharging of the SC due to the additional DCDC converter power consumption.

In our analysis and demonstration, a large SC $(0.1 \mathrm{~F})$ is chosen which is envisioned to power QPAR nodes with multiple OLSs and the control circuitry. The discharging time of the SC, which is defined as the time it takes for the voltage to drop from $4.6 \mathrm{~V}$ to $3.5 \mathrm{~V}$, was measured when the SC was used to drive one or two OLSs, and the results are shown in Figure 5. With one OLS as the load, the discharging time is $70 \mathrm{~s}$ (the OLS was continuously reconfigured). In addition, the discharging time is lowered to $35 \mathrm{~s}$ when the number of OLSs increases to two.

\section{Comparison of Different Remote Power Options}

3.1. Theoretical Analysis. As mentioned in Section 1, the QPAR node can be remotely powered using two schemes, namely, Direct Photovoltaic Power (Option 1) and Charged Supercapacitor Power (Option 2). To compare these two options, the following assumptions are made:

(i) Both options have the same optical link including $5 \mathrm{~km}$ long feeder fiber, band coupler/splitters, and power splitters. Thus both options have the same optical link loss, which can be calculated as listed in Table 2.

(ii) Both options have the same PPC, which has eight PDs in series and the responsivity of each PD is $0.9 \mathrm{~A} / \mathrm{W}$.

(iii) Both options have the same control circuit.

We define the following metrics to compare the two options.

3.1.1. System Energy Efficiency (SEE). The System Energy Efficiency of QPAR remote power is defined as follows:

$$
\begin{aligned}
\text { SEE }= & \frac{\text { Energy provided to QPAR }}{\text { Energy output by the power laser }} \\
= & \frac{\text { Energy fed into the PPC }}{\text { Energy output by the power laser }} \\
& \cdot \frac{\text { Energy provided to QPAR }}{\text { Energy fed into the PPC }} .
\end{aligned}
$$

TABLE 2: Optical link loss.

\begin{tabular}{lc}
\hline $\begin{array}{l}\text { Fiber attenuation } \\
(0.22 \mathrm{~dB} / \mathrm{km} \text { at } 1480 \mathrm{~nm})\end{array}$ & $2.2 \mathrm{~dB}$ \\
\hline $\begin{array}{l}\text { Band coupler/splitter } \\
\text { insertion loss }(0.4 \mathrm{~dB} / \text { each })\end{array}$ & $0.8 \mathrm{~dB}$ \\
\hline $\begin{array}{l}\text { Connector loss } \\
(0.3 \mathrm{~dB} / \text { each })\end{array}$ & $1.2 \mathrm{~dB}$ \\
\hline Power splitter excess loss & $1.0 \mathrm{~dB}$ \\
\hline Total loss & $5.2 \mathrm{~dB}$ \\
\hline E1 & $30 \%$ \\
\hline
\end{tabular}

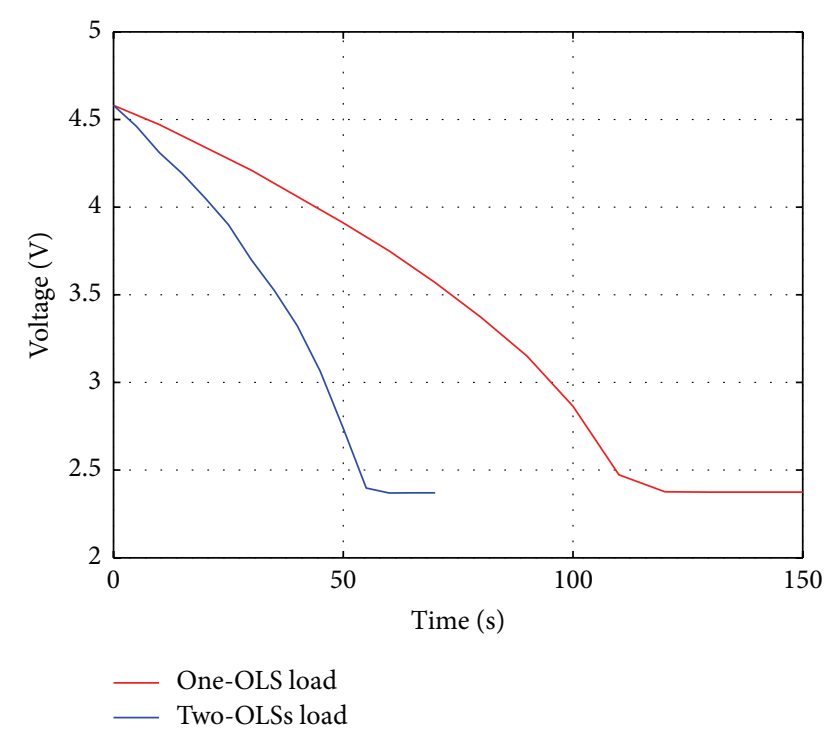

FIGURE 5: Discharging curves of a $0.1 \mathrm{~F}, 5 \mathrm{~V}$ SC with one-OLS and two-OLS load.

Let

$$
\begin{aligned}
& E_{1}=\frac{\text { Energy fed into the PPC }}{\text { Energy output by the power laser }}, \\
& E_{2}=\frac{\text { Energy provided to QPAR }}{\text { Energy ed into the PPC }} .
\end{aligned}
$$

Both options have the same $E_{1}=0.3$ (see Table 2), which is determined by the optical link loss. $E_{2}$ can be calculated using

$$
\begin{aligned}
& E_{2-} \text { Option } 1=\frac{P_{\mathrm{QPAR}} \cdot T_{\mathrm{rec}}}{N \cdot P_{\mathrm{in}} \cdot T_{\mathrm{rec}}}=V_{\mathrm{QPAR}} \cdot \frac{R_{p}}{N}, \\
& E_{2 \text { _Option } 2}=\frac{P_{\mathrm{QPAR}} \cdot T_{\mathrm{rec}}}{N \cdot P_{\mathrm{in}} \cdot T_{\text {charge }}}=\frac{V_{1}^{2}-V_{2}^{2}}{2 V_{1}} \cdot \frac{R_{p}}{N},
\end{aligned}
$$

where $V_{\mathrm{QPAR}}, V_{1}, V_{2}, N$, and $R_{P}$ are the average voltage of QPAR, charging and discharging stopping voltages, number of PDs, and the responsivity of PDs.

For Option 1, $E_{2}$ is proportional to the QPAR operation voltage, thus having a minimum value when $V_{\mathrm{QPAR}}=2.5 \mathrm{~V}$. For Option 2, $E_{2}$ depends on the charging and discharging stopping voltages, which have theoretical maximum value 


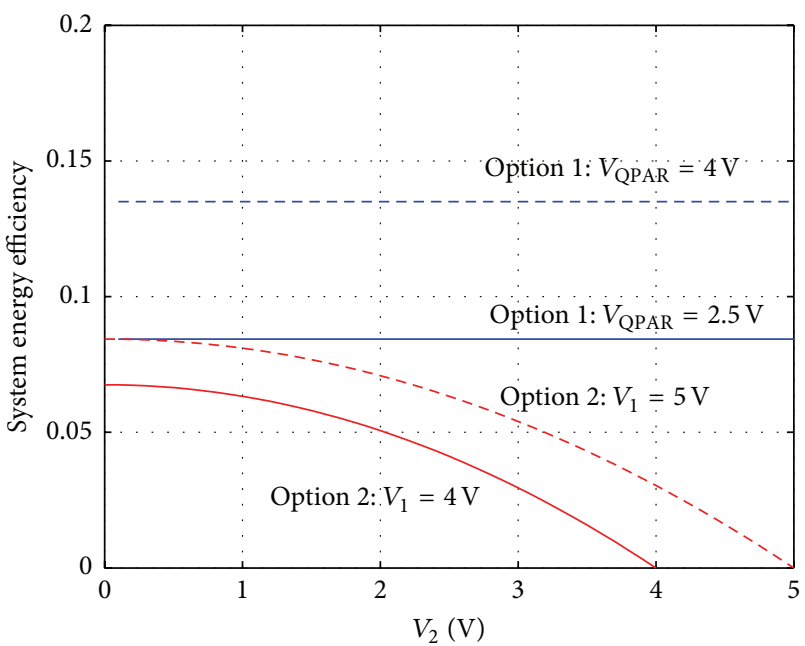

Figure 6: System Energy Efficiency (SEE).

when $V_{1}=5 \mathrm{~V}$ and $V_{2}=0 \mathrm{~V}$. As illustrated in Figure 6, the minimum SEE of Option 1 equals the maximum SEE of Option 2, where both are $8.4 \%$. Therefore, to power the same QPAR, Option 1 is more energy efficient, thus requiring less total energy.

3.1.2. Maximum Number of Simultaneously Powered OLSs $\left(N_{\text {OLS }}\right)$. The OLSs in QPAR can be powered either simultaneously or sequentially, or by using a combination of both. Simultaneous powering takes less time but requires higher instantaneous power. Here the two remote power mechanisms of QPAR are compared in terms of the maximum possible number of OLSs that can be driven simultaneously when the available power from the high power laser is the same. $N_{\text {OLS }}$ can be calculated using (6) and (7), respectively. For Option 1, $N_{\text {OLS }}$ depends on the current generated by the PPC. To power a large number of OLSs in parallel, high optical power is required to be fed into each PD in the PPC $\left(P_{\text {in }}\right)$. For Option 2, $N_{\text {OLS }}$ depends on the energy stored in SC as well as the percentage of energy used for discharging. As discussed in previous sections, $V_{1}=4.6 \mathrm{~V}$ and $V_{2}=3.5 \mathrm{~V}$ were selected. $N_{\text {OLS }}$ of Option 2 is also proportional to the ratio $T_{\text {charge }} / T_{\text {rec }}$, which is denoted by $T$. For a longer charging time, more energy can be stored in the SC to power more OLSs.

Similarly, a shorter reconfiguration time results in less energy being distributed to each OLS. Based on (6) and (7), the maximum number of OLSs that can be powered simultaneously $\left(N_{\text {OLS }}\right)$ for both options is shown in Figure 7:

$$
\begin{aligned}
& N_{\text {OLS }} \text { Option } 1=\frac{P_{\text {in }} \cdot R_{P}}{I_{\text {OLS }}}, \\
& N_{\text {OLS-Option } 2}=\frac{T_{\text {charge }}}{T_{\text {rec }}} \cdot \frac{V_{1}{ }^{2}-V_{2}^{2}}{2 V_{1} \cdot V_{\text {OLS }}} \cdot \frac{P_{\text {in }} \cdot R_{P}}{I_{\text {OLS }}} .
\end{aligned}
$$

It can be concluded from Figure 7 that as long as $T$ is larger than 4 , that is, the charging time is at least four times as long as the reconfiguration time, Option 2 can power more

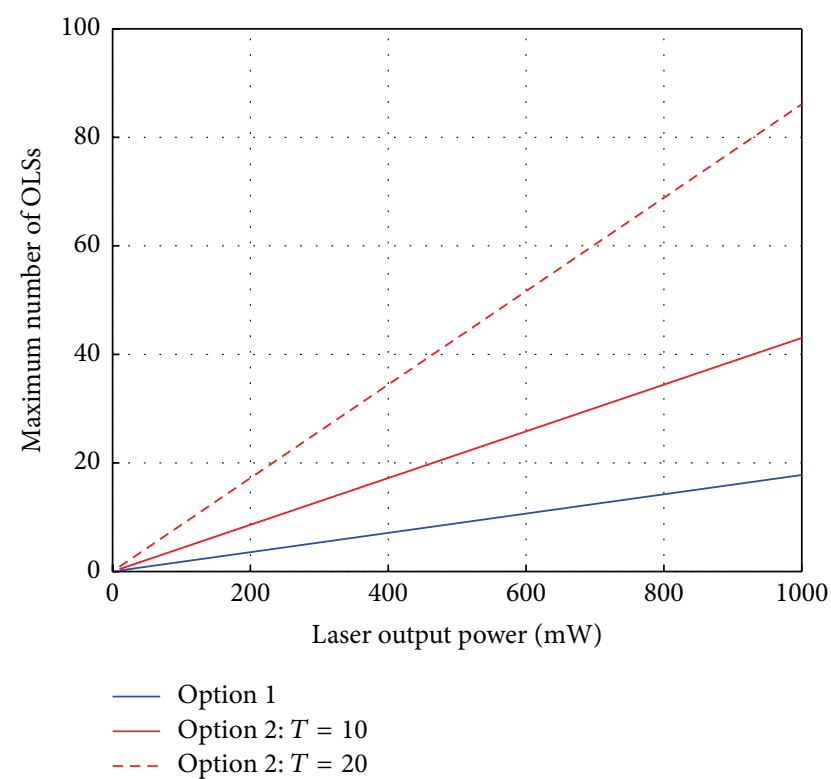

FIGURE 7: Maximum number of OLSs powered simultaneously $\left(N_{\text {OLS }}\right)$.

OLSs simultaneously with the same total power provided by the high power laser. $T$ is typically larger than 4; therefore, Option 2 is more suitable for a high dimension QPAR which has a larger number of OLSs, especially when the optical power that can be transmitted to the $\mathrm{RN}$ is limited. If a high dimension QPAR needs to be powered using Option 1, the OLSs should be powered sequentially at the cost of a longer QPAR reconfiguration time.

3.1.3. Total Time to Complete the Reconfiguration ( $\left.T_{\text {total }}\right)$. To compare $T_{\text {total }}$ of Option 1 and Option 2, we made two more assumptions as follows:

(i) Both options are used to power the same QPAR with NUM OLSs.

(ii) Both options have the same total available power, which is only enough to power NUM OLSs simultaneously using Option 2; that is, NUM = $N_{\text {OLS }}($ Option 2).

Therefore, to power NUM OLSs using Option 1, they should be divided into subgroups and the subgroups are powered in sequence. Assume that each subgroup contains $N_{\text {OLS }}$ (Option 1) OLSs. The number of subgroups $(M)$ is the ratio between $N_{\text {OLS }}\left(\right.$ Option 2) and $N_{\text {OLS }}$ (Option 1), which can be obtained by using (6) and (7):

$$
M=\frac{N_{\text {OLS }}(\text { Option 2) }}{N_{\text {OLS }}(\text { Option 1) }}=\frac{T_{\text {charge }}}{T_{\text {rec }}} \cdot \frac{V_{1}^{2}-V_{2}^{2}}{2 V_{1} \cdot V_{\text {OLS }}} .
$$

We can prove that the second term in (8) is smaller than 1 ; therefore, $M$ is smaller than $T_{\text {charge }} / T_{\text {rec }}$.

For Option 1, the time it takes to complete the reconfiguration is the sum of the reconfiguration time of $M$ subgroups, which is less than $T_{\text {charge }}$ (see (9)). For Option 2, all the NUM 
TABLE 3: System Energy Efficiency comparison.

\begin{tabular}{lcccc}
\hline & \multicolumn{2}{c}{ Option 1: Direct Photovoltaic Power } & \multicolumn{2}{c}{ Option 2: Charged Supercapacitor Power } \\
& Theoretical & Experimental & Theoretical & Experimental \\
\hline E1 & $30 \%$ & $20 \%$ & $30 \%$ & $20 \%$ \\
E2 & $27.5 \%$ & $25 \%$ & $7.2 \%$ & $7 \%$ \\
SEE & $8.3 \%$ & $5 \%$ & $2.2 \%$ & $1.4 \%$ \\
\hline
\end{tabular}

OLSs can be powered simultaneously in $T_{\text {rec }}$. However, the total time to complete the reconfiguration is the sum of $T_{\text {charge }}$ and $T_{\text {rec }}$ (see (10)), which is longer than using Option 1:

$$
\begin{aligned}
& T_{\text {total }}(\text { Option } 1)=M \cdot T_{\text {rec }}<\frac{T_{\text {charge }}}{T_{\text {rec }}} \cdot T_{\text {rec }}=T_{\text {charge }}, \\
& T_{\text {total }}(\text { Option } 2)=T_{\text {charge }}+T_{\text {rec }}
\end{aligned}
$$

3.2. Experimental Results. Experiments were carried out to investigate both QPAR remote power options. The results on SEE are listed in Table 3. In both options, the output power of the pump laser was $100 \mathrm{~mW}(20 \mathrm{dBm})$, and the insertion loss of the optical path (including the band coupler/splitter, fiber, connectors, and power splitters) was about $16 \mathrm{~dB}$. Therefore, $4 \mathrm{dBm}$ optical power was fed into each $\mathrm{PD}\left(P_{\text {in }}\right)$ of the $1 \times 8$ $\mathrm{PD}$ array.

Using Option 1, a minimum $4 \mathrm{dBm} P_{\text {in }}$ was required to power one $2 \times 2$ OLS, which worked at the minimum power consumption point $(2.5 \mathrm{~V} / 2 \mathrm{~mA} / 5 \mathrm{~mW})$. The overall system conversion efficiency is $5 \%$ [3].

Using Option 2, the same $4 \mathrm{dBm}$ optical feed charged a $0.1 \mathrm{~F} / 5 \mathrm{~V} \mathrm{SC}$ from $0 \mathrm{~V}$ to $4.6 \mathrm{~V}$ in $310 \mathrm{~s}$. The charged SC was capable of powering the same OLS for $70 \mathrm{~s}$ before the SC voltage dropped below $3.5 \mathrm{~V}$. The overall system conversion efficiency is $1.4 \%$.

Although Option 1 has a higher SEE, the same optical power is only enough to power one $2 \times 2$ OLS. To power two $2 \times 2$ OLSs simultaneously, at least $9 \mathrm{dBm}$ fed into each PD is required [3]. On the other hand, the number of simultaneously powered OLSs using Option 2 is not limited by the optical power fed into each PD. As long as the extra charging time is acceptable, Option 2 is capable of powering a large number of OLSs.

In summary, both options have pros and cons in different applications. With a higher SEE and shorter $T_{\text {total }}$, Option 1 is more advantageous when the remote power application is energy constraint or time constraint, while Option 2 wins when the available optical power is the bottleneck. Therefore, these two schemes should be selected according to application requirements: Option 1 should be used in small dimension QPARs or sequential reconfiguration of OLSs in QPARs, while Option 2, with the potential of powering more OLSs simultaneously, is a better solution for high dimension QAPRs.

\section{Remote Control of QPAR}

The control signal of the QPAR, which decides the states of OLSs, can be also remotely and optically transmitted, converted by another PPC and further decoded by the control circuit. In this section, the general architecture of QPAR control circuit is proposed and the implementation of a control circuit that is capable of controlling a QPAR with eight OLSs is demonstrated.

4.1. Architecture of QPAR Control Circuit. Figure 8 shows the general architecture of QPAR control circuit, which has four function units.

(1) Analog Switch Unit. This unit contains two stages of cascaded analog switches. The first switch (SW1) is kept off during the SC charging process. During reconfiguration, SW1 is turned on so that the SC is connected as the power supply for the control circuit. Though designed for Option 2, the same control circuit can be used in Option 1, with SW1 being removed since the power supply is controlled by the power laser in the CO directly.

The second stage switch (SW2) connects the power supply to the subgroup of OLSs in QPAR that needs to be powered. This not only avoids providing power to the OLSs whose states are unchanged to reduce the total energy required in the SC, but also simplifies the control circuit since different subgroups share the same electrical devices. Both switches SW1 and SW2 should have small resistance and fast switching properties.

(2) SW1 Control Unit. The function of this unit includes detecting the arrival of control signals so as to turn on SW1 and detecting the completion of the reconfiguration so as to turn off SW1. This unit is always powered on; thereby it must have very small current and power consumption.

(3) Clock Generator Unit. When powered on, this unit generates the clock at a certain frequency, which is used for synchronization. Requirements for this unit are low power consumption, reliability, and high-quality synchronization.

(4) Control Signal Decoding Unit. This unit is a "serial in parallel out" system that can extract the status information of QPAR from the control signal and map it onto the designated subgroup of OLSs. Besides low power consumption, this unit should also be reliable and scalable especially for high dimension QPARs.

4.2. Control Circuit Demonstration. Based on the four function units, a control circuit as shown in Figure 9 was designed and demonstrated which had four subgroups and was capable of powering two OLSs simultaneously in each subgroup. 


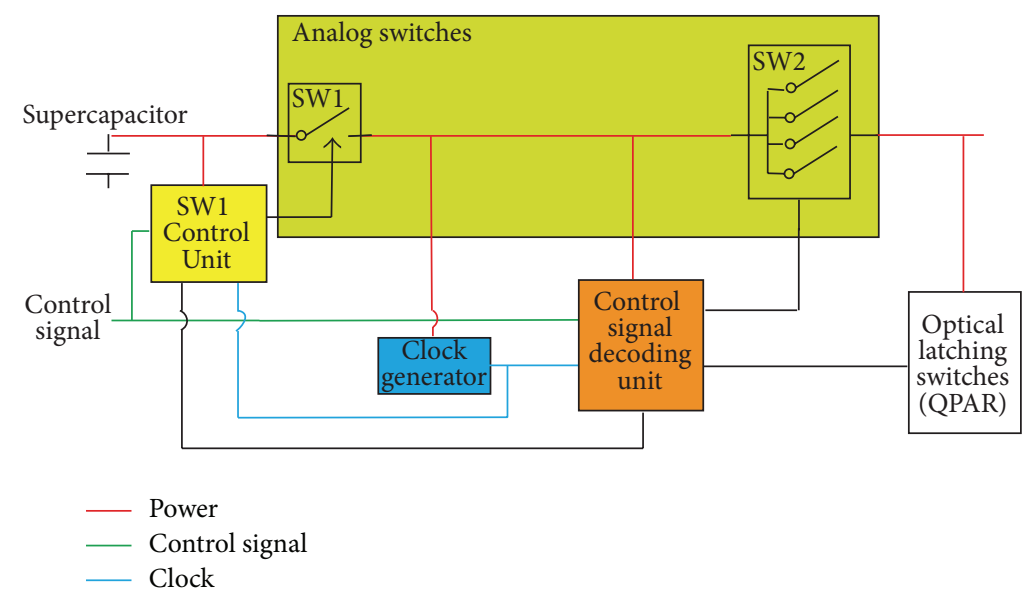

FIGURE 8: General architecture of QPAR control circuit.

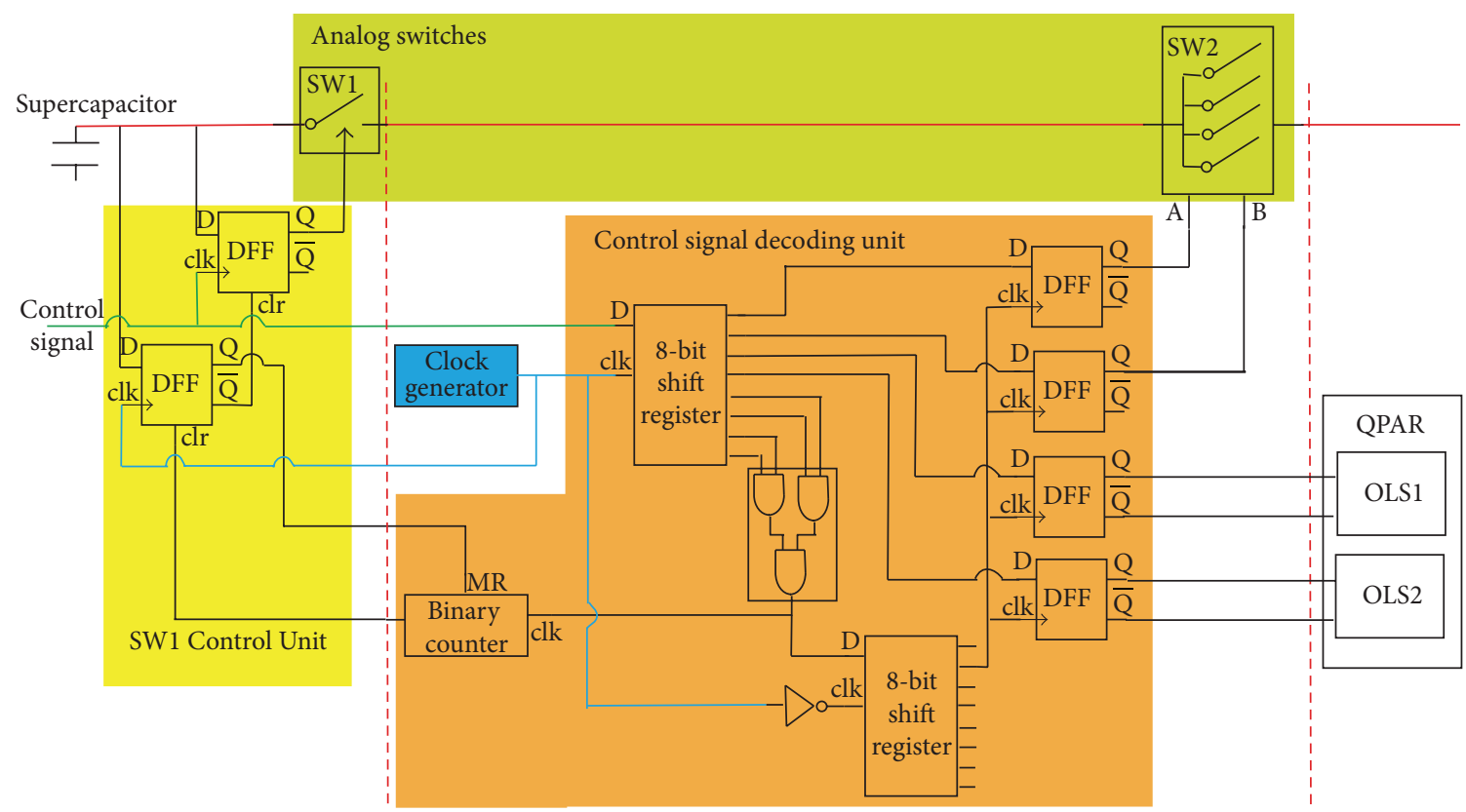

Figure 9: Control circuit example.

An example of the control signal, which is the input of designed control circuit, is shown in Figure 9. The control signal consists of starting bits, synchronization bits, and QPAR state bits. The first up-edge of the starting bits is detected by the SW1 Control Unit to turn on SW1 so that the control circuit is powered on. The synchronization bits "1111" are detected by the control signal decoding unit to map the QPAR state " 1010 " to the outputs of the four D-type flipflops, which will remain there unless the SW1 is turned off and the power supply of the control circuit is cut off. There are four QPAR state bits, "1010" in the example, where the first two digits are to select the subgroup of OLSs and the last two digits correspond to the bar/cross states of the two OLSs in each subgroup. The timing diagram is plotted in Figure 10. In practice, the control signal may be repeated several times to guarantee the correct reconfiguration of the QPAR state.
In the current design, one straightforward way to scale up the control circuit is to enlarge the number of OLSs in each subgroup. However, the size of shift register, the number of D-type flip-flops, and the length of control signal all scale up quickly. The use of SW2 can help mitigate this problem, since the control circuit can be scaled up by simply adding more branches in SW2, thus increasing the number of subgroups. It should be noted that current design of the control circuit has not been optimized in terms of power consumption. Lower power digital chips can be used for more versatile operations, and more robust control coding is also subject to future research.

4.3. Remote Reconfiguration of a $1 \times 2 \times 2 Q P A R$. The remote power and control of a $1 \times 2 \times 2$ QPAR using a SC was experimentally demonstrated using the setup as shown in 


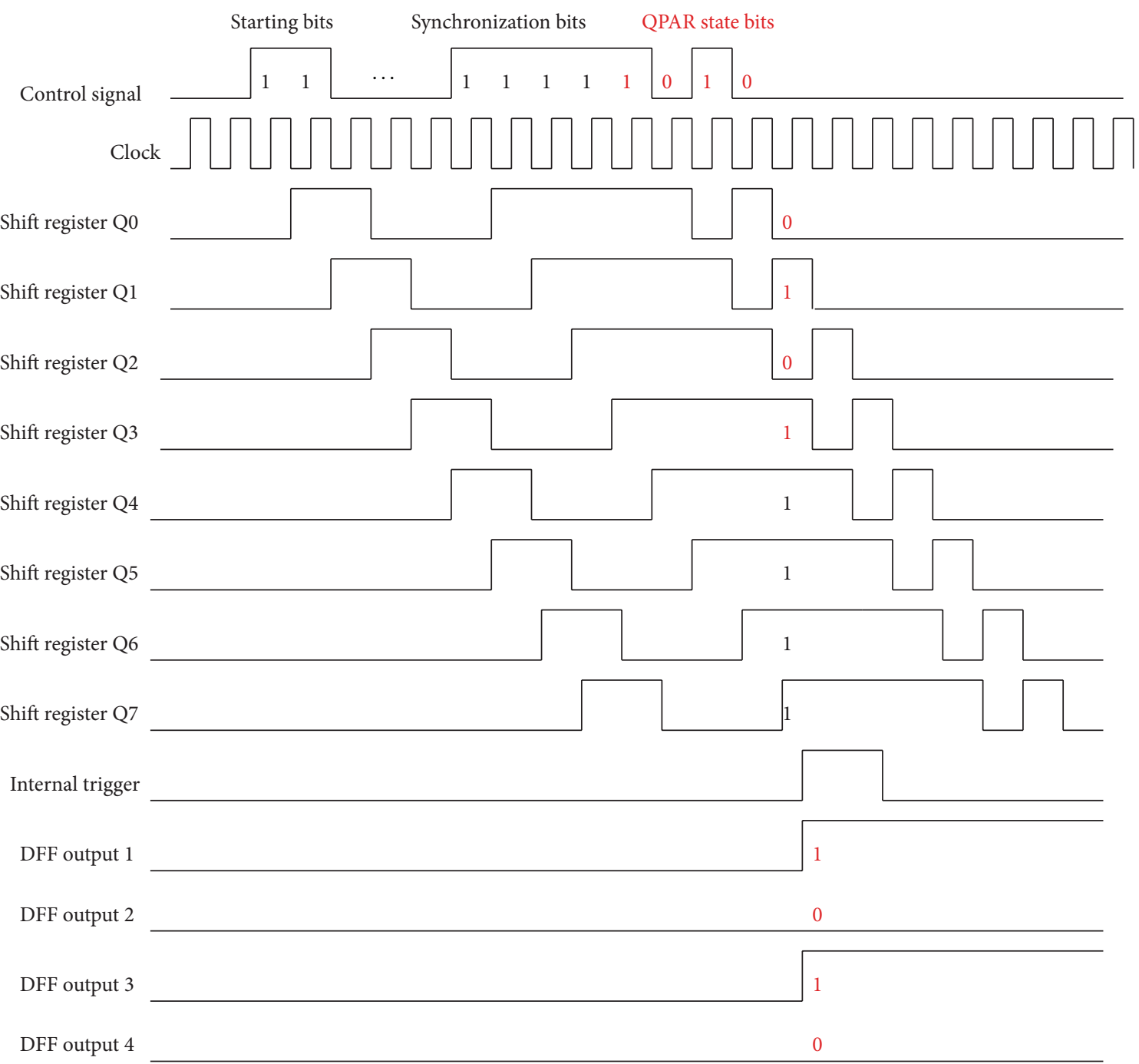

FIgURE 10: Timing diagram of the control signal decoding unit.
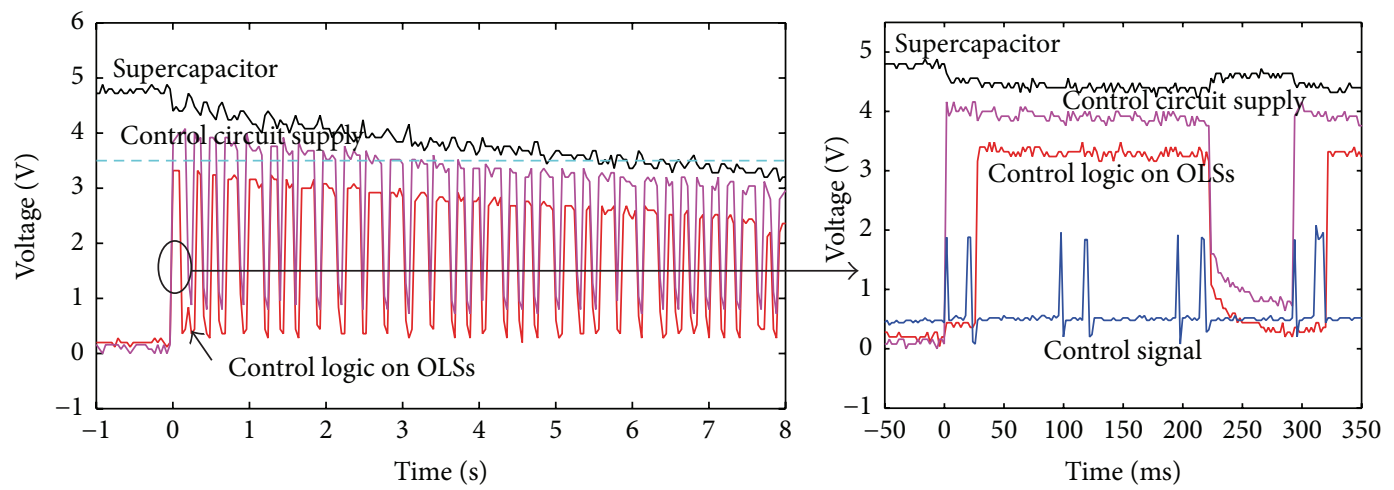

FIgURE 11: Discharging of the SC with the control circuit and OLS load.

Figure 13. The power laser was operated at the $1480 \mathrm{~nm}$ wavelength, while the control light and payload signal was transmitted at $1553 \mathrm{~nm}$ and $1595 \mathrm{~nm}$, respectively. The 1564$1620 \mathrm{~nm} / 1504-1557 \mathrm{~nm} / 1484-1497 \mathrm{~nm}$ band coupler/splitter was used to couple/decouple the power, control, and signal lights into/out of a $5 \mathrm{~km}$ single mode feeder fiber. Once decoupled from fiber, the power light was split by a $1 \times 8$ power splitter, and each output port was connected to a PD. The $1 \times 8 \mathrm{PD}$ array was used to charge a $0.1 \mathrm{~F} / 5 \mathrm{~V}$ SC. The control light was split by a $1 \times 4$ power splitter, converted by 


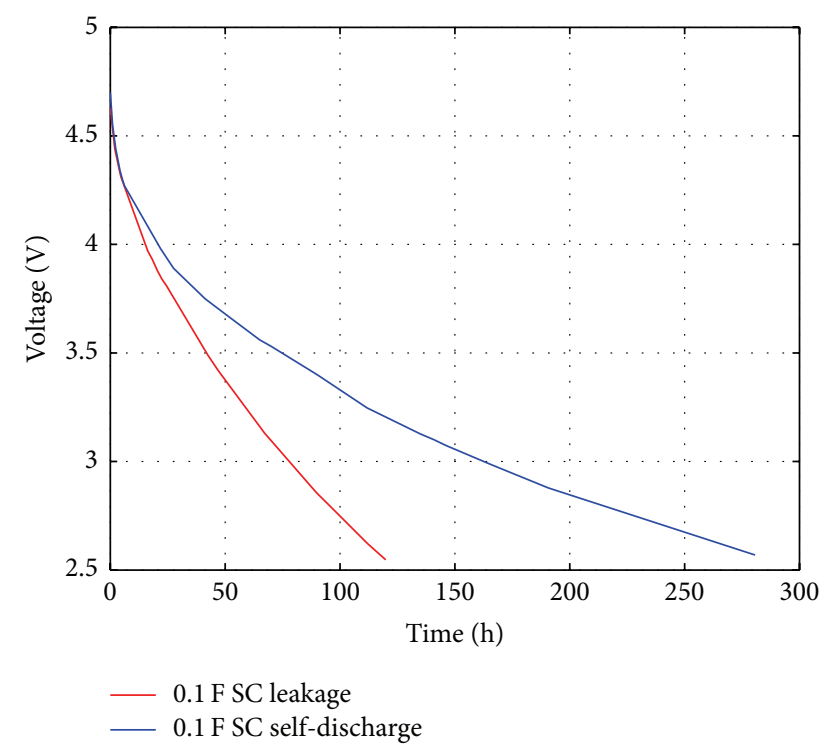

FIGURE 12: Leakage and self-discharge of a $0.1 \mathrm{~F}$ SC.

the $1 \times 4 \mathrm{PD}$ array, and then injected into the control circuit, whose detailed structure is shown in Figure 7 . In the $1 \times 2 \times 2$ QPAR, two $2 \times 2$ OLSs combined with one $3 \mathrm{~dB}$ coupler can achieve three states at the two output ports $\left(P_{\text {output } 1}, P_{\text {output } 2}\right)$ : $(P, 0),(0, P)$ and $(1 / 2 P, 1 / 2 P)$, where $P$ is the total input power of QPAR.

During the charging process, the SC is charged to $4.6 \mathrm{~V}$ in $310 \mathrm{~s}$ with $20 \mathrm{dBm}$ output power from the power laser. No power penalty of the payload signal was observed when the power light was transmitted together with the payload signal in the same fiber [3].

During the discharging process, an arbitrary function generator was programmed to generate the control signal, which was modulated onto the $1553 \mathrm{~nm}$ optical channel. After being converted to the electrical domain by a $1 \times 4$ InGaAs PD array in QPAR, the encoded switching instructions were extracted by the control circuit to toggle the states of the OLSs. To ensure the correctness of control process, the same control signal was repeated for three cycles. In each cycle, the control circuit and power supply to the OLSs remained on for $200 \mathrm{~ms}$ and off for $100 \mathrm{~ms}$. In such configuration, there were 24 consecutive cycles before the SC voltage dropped below $3.5 \mathrm{~V}$, as illustrated in Figure 11. The energy consumption of each reconfiguration cycle can be calculated to be $18.6 \mathrm{~mJ}$.

As illustrated in Figure 12, the leakage of SC was characterized when it was connected with the control circuit without any reconfiguration operation. When there is no reconfiguration request, it takes 40 hours for the SC voltage to drop below $3.5 \mathrm{~V}$. Therefore, within approximately 40 hours, another reconfiguration can be operated without charging the $\mathrm{SC}$ again. This leakage rate is slow enough compared to the charging time, which is in the order of several minutes. We also characterized the self-discharge of the SC as shown in Figure 12. It takes 70 hours for the SC to self-discharge to $3.5 \mathrm{~V}$ when no load is connected. This places an upper limit of the leakage time with different control circuit design.
In Figure 14, we capture the change of the optical signal at output 1 of the QPAR node under two reconfigurations: from $(0, P)$ to $(1 / 2 P, 1 / 2 P)$ and from $(1 / 2 P, 1 / 2 P)$ to $(P, 0)$. It is clear that the $1 \times 2 \times 2$ QPAR can be reliably reconfigured using the proposed remote power and control system. Though equipped with a $1 \times 2 \times 2$ QPAR, our testbed can support the reconfiguration of a higher dimension QPAR with eight OLSs since a $1 \times 4$ analog switch is used as SW2 in the control circuit.

\section{Conclusion}

A QPAR remote power mechanism using the energy stored in a supercapacitor at the RN has been proposed and demonstrated. The supercapacitor has been charged by remotely transmitted optical power from the CO. This Charged Supercapacitor Power scheme (Option 2) has been compared with the previously demonstrated Direct Photovoltaic Power solution (Option 1). It has been shown that Option 1 can guarantee that the RN is fully passive and has no energy leakage when there is no reconfiguration, while it is only suitable for small dimension QPARs due to the limited power provided. On the other hand, Option 2 has been shown to be capable of powering a larger number of OLSs simultaneously, thus making the remote power of high dimension QPARs possible. With the proposed remote power schemes, the flexible power/wavelength management and graceful system upgrade enabled by QPARs can be realized without having local power supplies and on-site reconfiguration at the RN. However, there is additional cost due to the additional components for remote power, such as high power lasers. Since the additional components are only added in either the $\mathrm{CO}$ or RN, the cost is shared by multiple end users. In addition, with the quasi-passive characteristics, remote powering of QPARs is only needed during reconfigurations. Given the low network reconfiguration frequency, remotely powered QPARs preserve the passive nature and maintain high energy efficiency of the network.

A control circuit has also been designed to remotely reconfigure QPARs by transmitting control signals from the CO. Based on Option 2, a remote power and control system has been demonstrated which can reconfigure a QPAR node with eight OLSs divided into four subgroups, without having any local power supply. It has been shown that a $0.1 \mathrm{~F} / 5 \mathrm{~V} \mathrm{SC}$, which is charged to $4.6 \mathrm{~V}$, can power a $1 \times 2 \times 2$ QPAR for $6 \mathrm{~s}$. With $200 \mathrm{~ms}$ of each reconfiguration, the implemented remote power system has been able to support at least 24 consecutive reconfigurations before the SC needs to be recharged. It has also been shown that the charging cycle takes $310 \mathrm{~s}$ with $13 \mathrm{dBm}$ optical feed into the $1 \times 8 \mathrm{PD}$ array, which is negligible compared to the leakage time of the system (in the order of tens of hours).

\section{Conflict of Interests}

The authors declare that there is no conflict of interests regarding the publication of this paper. 


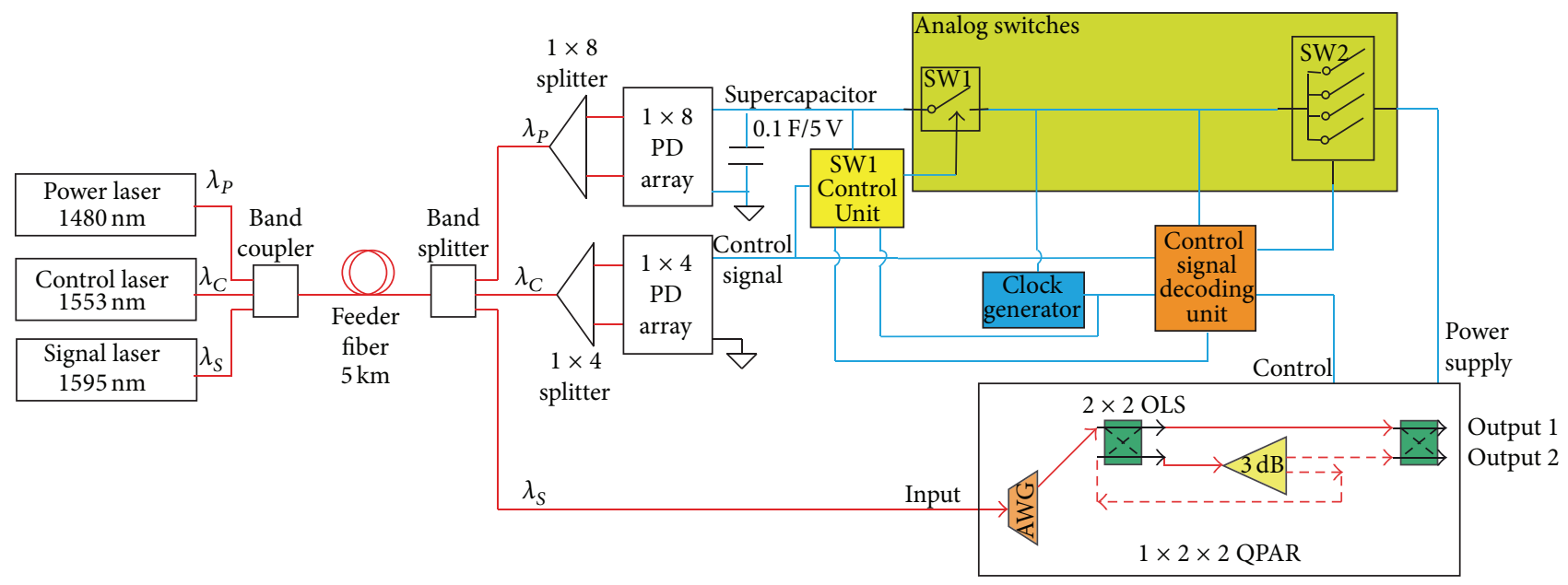

FIGURE 13: Testbed of the $1 \times 2 \times 2$ QPAR remote power and reconfiguration.

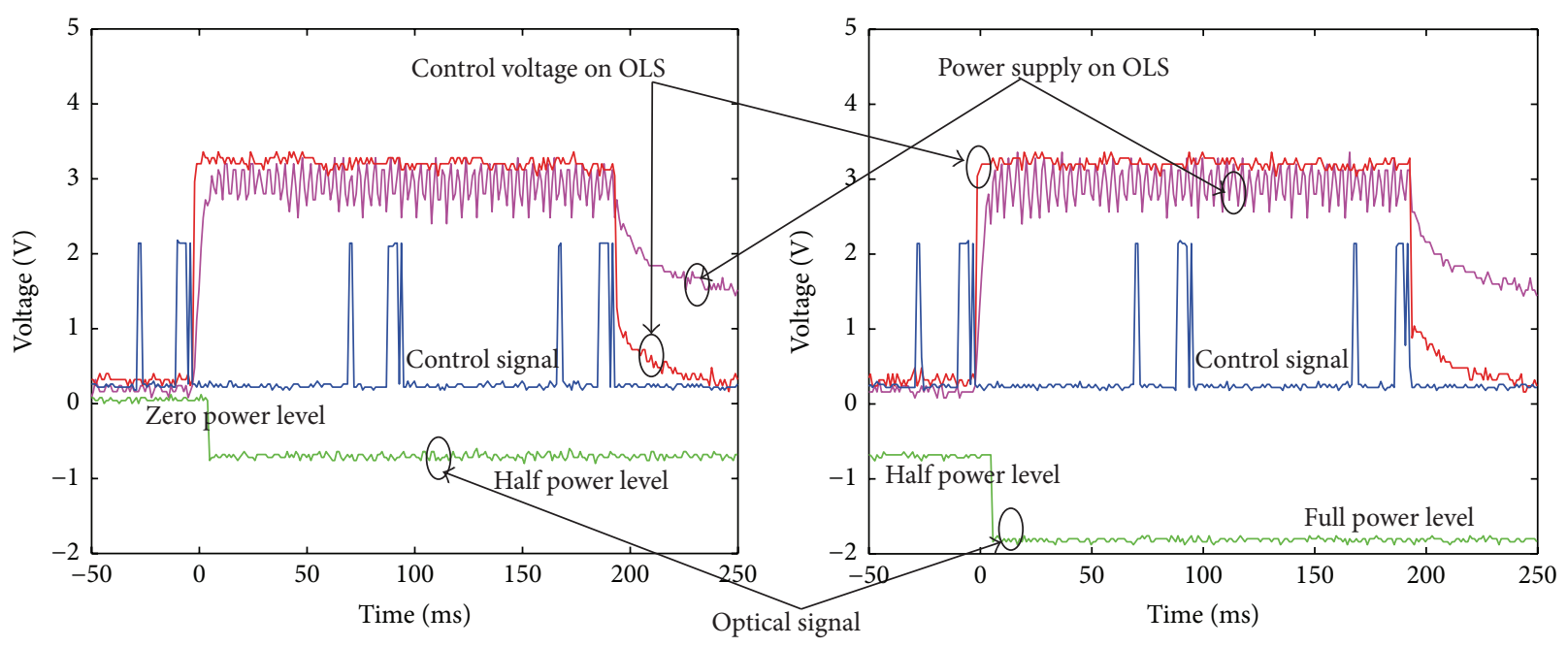

FIGURE 14: Experimental results of the $1 \times 2 \times 2$ QPAR remote reconfiguration.

\section{Acknowledgments}

This work has been partially funded by the Center for Integrated Systems, Stanford University. The authors would like to thank Sercalo for providing OLSs for their experiments.

\section{References}

[1] Y. Bi, J. Jin, A. R. Dhaini, and L. G. Kazovsky, "QPAR: a quasi-passive reconfigurable green node for dynamic resource management in optical access networks," Journal of Lightwave Technology, vol. 32, no. 6, Article ID 6691901, pp. 1104-1115, 2014.

[2] Y. Bi, J. Jin, A. Dhaini, and L. G. Kazovsky, "Experimental demonstration of a high-dimension quasi-passive reconfigurable (QPAR) node," in Proceedings of the Conference on Lasers and Electro-Optics (CLEO '14), paper STu1J.7, Optical Society of America, San Jose, Calif, USA, June 2014.

[3] Y. Bi, J. Jin, and L. G. Kazovsky, "First experimental demonstration of a remotely powered quasi-passive reconfigurable node," IEEE Photonics Technology Letters, vol. 27, no. 9, pp. 990-993, 2015.
[4] H. Ramanitra, P. Chanclou, Z. Belfqih, M. Moignard, H. Le Bras, and D. Schumacher, "Scalable and multi-service passive optical access infrastructure using variable optical splitters," in Proceedings of the Optical Fiber Communication Conference, and the National Fiber Optic Engineers Conference (OFC '06), IEEE, Anaheim, Calif, USA, March 2006.

[5] B. Schrenk, A. Poppe, M. Stierle, and H. Leopold, "Passive optical switching engine for flexible metro-access," in Proceedings of the European Conference on Optical Communication (ECOC '14), pp. 1-3, Cannes, France, September 2014.

[6] Supercapacitor, http://batteryuniversity.com/learn/article/whats _the_role_of_the_supercapacitor.

[7] M. M. Mahmoud, “Transient analysis of a PV power generator charging a capacitor for measurement of the I-V characteristics," Renewable Energy, vol. 31, no. 13, pp. 2198-2206, 2006.

[8] $2 \times 2$ MEMS OLS Data Sheet, http://www.sercalo.com/products/ pdfs/SL2x2.pdf. 


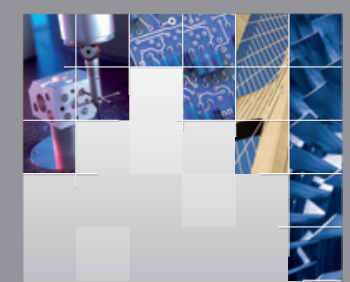

\section{Enfincering}
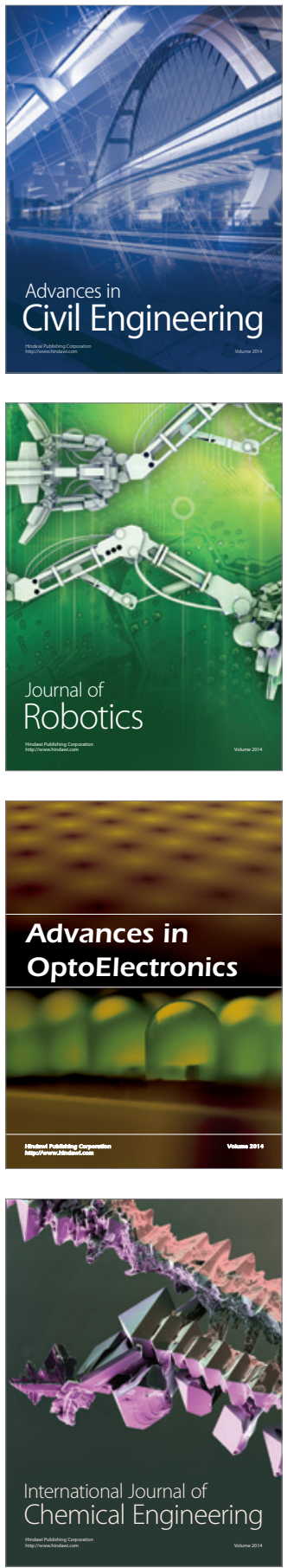

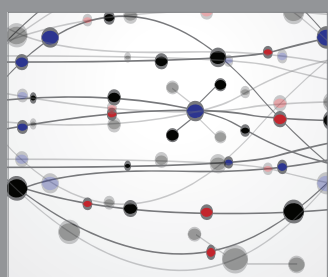

The Scientific World Journal

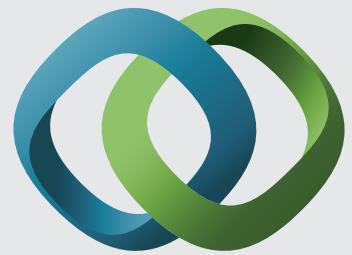

\section{Hindawi}

Submit your manuscripts at

http://www.hindawi.com
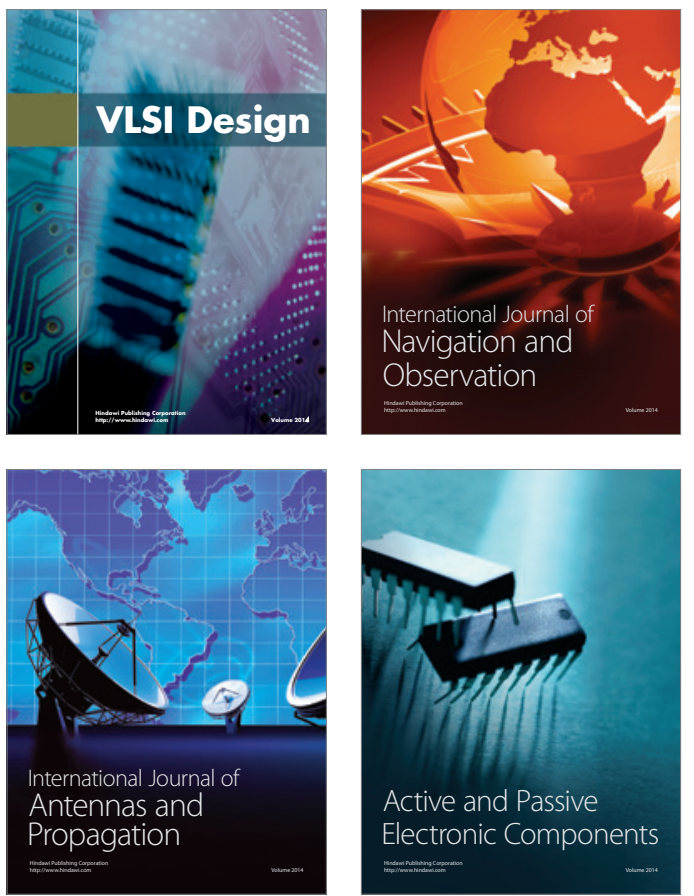
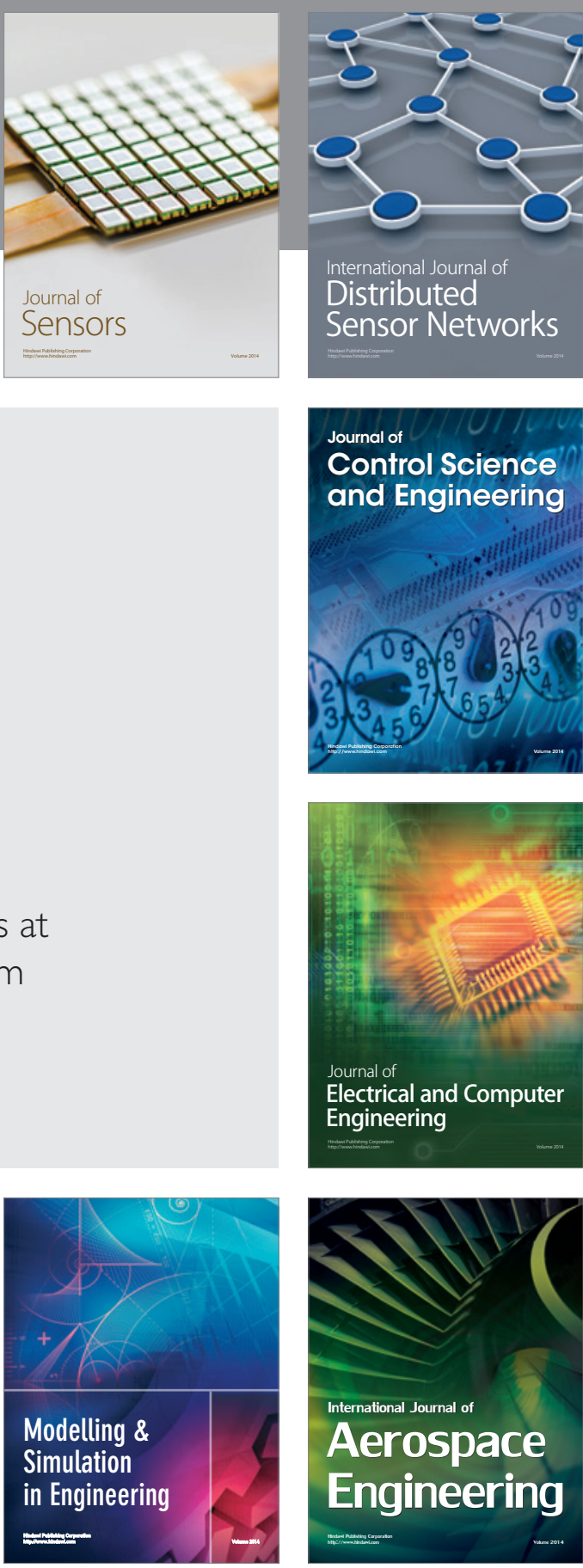

International Journal of

Distributed

Sensor Networks

Journal of

Control Science

and Engineering
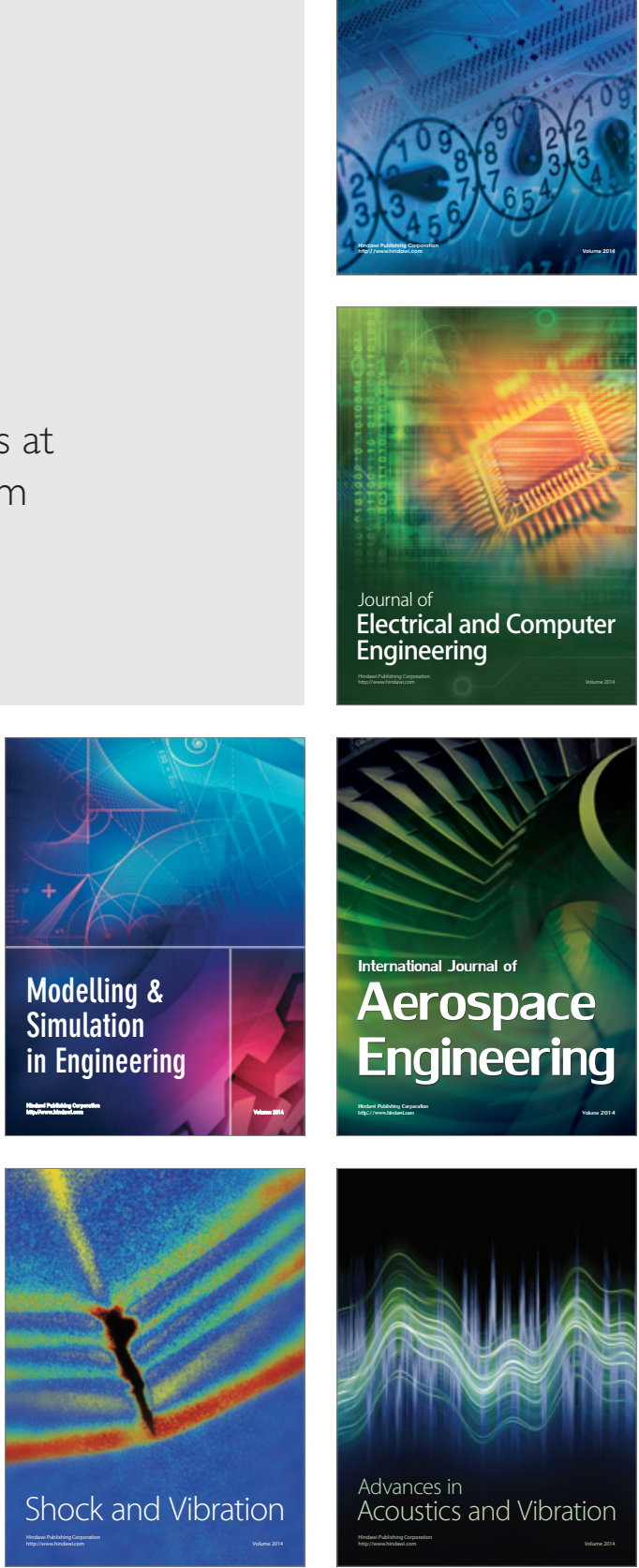